\title{
Growth differentiating factor-15 (GDF-15): A potential biomarker and therapeutic target for cancer-associated weight loss
}

\author{
LORENA LERNER $^{1}$, JENO GYURIS ${ }^{1}$, RICHARD NICOLETTI ${ }^{1}$, \\ JAMES GIFFORD $^{1}$, BRIAN KRIEGER ${ }^{1}$ and AMINAH JATOI ${ }^{2}$ \\ ${ }^{1}$ AVEO Pharmaceuticals, Cambridge, MA 02142; ${ }^{2}$ Department of Oncology, Mayo Clinic, Rochester, MN 55905, USA
}

Received September 24, 2015; Accepted June 2, 2016

DOI: $10.3892 / \mathrm{ol} .2016 .5183$

\begin{abstract}
Growth differentiating factor-15 (GDF-15), also known as macrophage inhibiting factor-1, is a member of the transforming growth factor- $\beta$ superfamily, which has been implicated in cancer-associated weight loss. The present study investigated the association between cancer-associated weight loss and plasma GDF-15 concentration, as well as other biomarkers, in patients with metastatic lung or exocrine pancreatic cancer. A total of 218 patients were enrolled over a 1 -year period. The patient cohort included 152 patients with incurable lung cancer and 66 patients with incurable pancreatic cancer. Of the 218 patients, $98(45 \%)$ reported $>5 \%$ weight loss, $62(28 \%)$ reported $\leq 5 \%$ weight loss and $58(27 \%)$ reported no weight loss in the 6 months prior to diagnosis. In lung cancer patients, higher circulating GDF-15 levels were significantly associated with weight loss; lung cancer patients who reported $>5 \%$ weight loss $(n=56)$ were found to exhibit twice the circulating concentration of GDF-15 compared with those that exhibited no weight loss $(\mathrm{n}=48)(\mathrm{P}<0.0001)$. Additional mediators, including Activin A, interleukin (IL)-12, vascular endothelial growth factor $\mathrm{A}, \mathrm{IL}-1$ receptor $\alpha$, eotaxin and platelet derived growth factor-BB, were also associated with weight loss; however, the associations were not as strong. In pancreatic cancer patients, no association between GDF-15 levels and weight loss was identified. However, higher circulating GDF-15 levels were consistently associated with poor survival in univariate [hazard ratio (HR), 1.13; 95\% confidence interval (CI), 1.02-1.23; $\mathrm{P}=0.016]$ and multivariate [HR, 1.1; 95\% CI, 1.02-1.24; $\mathrm{P}=0.03$ ] analysis, respectively. Thus, GDF-15 requires further study as a biomarker and potential therapeutic target in cancer-associated weight loss, particularly in lung cancer patients.
\end{abstract}

Correspondence to: Dr Aminah Jatoi, Department of Oncology, Mayo Clinic, 200 First Street SW, Rochester, MN 55905, USA

E-mail: jatoi.aminah@mayo.edu

Key words: weight loss, cancer, growth differentiating factor-15, therapeutic target

\section{Introduction}

Cancer-associated weight loss occurs in $60-80 \%$ of patients with incurable cancer and leads to debility and shortened survival (1). This weight loss is the result of poor oral intake and changes in basal metabolism; however, by contrast to starvation, it is irreversible with caloric supplementation (1). Previous studies have also demonstrated that this weight loss is refractory to pharmacological interventions $(2,3)$. Although multiple biological mediators, including pro-inflammatory cytokines, neuroendocrine hormones, lipid-mobilizing factors and alterations in intermediary metabolism, have been implicated in the pathobiology of cancer-associated weight loss, it is hypothesized that numerous key mediators remain unidentified and uncharacterized at present (4). The identification of certain key mediators may present potential therapeutic targets and lead to the development of drugs that improve outcomes in cancer patients exhibiting weight loss and may also provide biomarkers to assess the early outcomes of future palliative trials.

Growth differentiating factor-15 (GDF-15), also known as macrophage inhibiting factor-1, is a member of the transforming growth factor- $\beta$ superfamily that is involved in myocyte remodeling, cardiovascular disease, dysfunctional erythropoiesis, general weight loss and early carcinogenesis (5-7). At least three clinical studies have identified an association between GDF-15 and cancer-associated weight loss (8-10). Firstly, Johnen et al (8) examined GDF-15 levels in 36 prostate cancer patients with and without weight loss and observed that circulating GDF-15 concentrations were significantly higher in patients that exhibited weight loss. Secondly, Vigano et al (9) examined 207 cancer patients and observed that serum GDF-15 levels were directly correlated with worse Patient-Generated Subjective Global Assessment scores. When evaluated by quartile, higher serum GDF-15 levels were also associated with poor survival. Thirdly, Lerner et al (10) preliminarily examined 62 cancer patients with weight loss, 72 cancer patients with no weight loss and 64 non-cancer patients and reported that serum GDF-15 concentrations in cancer patients with weight loss were significantly higher than patients with no weight loss and non-cancer patients $(\mathrm{P}<0.001)$. Similarly, GDF-15 concentration was significantly and inversely correlated with lean body mass $(\mathrm{P}<0.001)$ and grip strength $(\mathrm{P}=0.007)(10)$. These data suggest that GDF-15 may 
be a relevant biomarker in patients with cancer-associated weight loss and thus, investigation of GDF-15 as a therapeutic target must be considered.

The present study investigated the association between GDF-15 levels and weight loss in patients with incurable cancer. By contrast to previous studies, this study aimed to incorporate the consensus definition of cancer-associated weight loss as described by Fearon et al (11). In addition, the present study focused specifically on patients with incurable lung cancer and exocrine pancreatic cancer using a relatively large sample size in comparison to previous studies. The larger sample size was selected in order to reduce heterogeneity within the study population, thereby enabling reliable evaluation of the association between circulating GDF-15 concentration and survival. Furthermore, the present study analyzed additional inflammatory markers and potential mediators of cancer-associated weight loss with the aim of providing benchmark data that might put findings related to GDF-15 in greater clinical context. Overall, the aim of the present study was to further explore GDF-15 as a biomarker for cancer-associated weight loss and to identify a potential therapeutic target to palliate cancer-associated weight loss.

\section{Materials and methods}

Patient eligibility. The study was approved by the Institutional Review Board at the Mayo Clinic (Rochester, MN, USA). Patients that met the following eligibility criteria were selected for study enrollment: i) $\geq 18$ years of age; ii) able and willing to provide informed consent; iii) diagnosed with incurable cancer of the lung or exocrine pancreas; iv) no chemotherapy administered in the 7 days prior to enrollment; and v) no peripheral edema or abdominal ascites.

Patient enrollment and follow up. A study coordinator identified eligible patients and subsequently obtained written consent. A total of 218 patients were enrolled, including 152 patients with incurable lung cancer and 66 patients with incurable pancreatic cancer. Patients provided information regarding weight loss in the 6 months prior to enrollment and blood samples were obtained from each patient. The patients were classified into the following groups: $>5 \%$ weight loss; $\leq 5 \%$ weight loss; and no weight loss (11).

The medical records of all patients were reviewed for demographic data and other relevant clinical information. Patients were followed-up until the date of mortality or last follow-up with censoring.

Handling and assaying blood samples. Plasma samples were assigned a unique code with no clinically identifying information. Samples were centrifuged for separation of plasma, which was stored at $-80^{\circ} \mathrm{C}$ prior to use. All samples were sent to AVEO Pharmaceuticals (Cambridge, MA, USA) for subsequent analyses.

Biomarkers were measured in a blinded manner; patient clinical data, including degree of weight loss, were unavailable to laboratory staff. Individual ELISA kits (R\&D Systems, Inc., Minneapolis, MN, USA) were used to measure GDF-15 and Activin A concentration. The concentrations of the remaining markers were measured using the Bio-Plex Pro ${ }^{\mathrm{TM}}$
Table I. Patient demographics of 218 patients enrolled in the study.

\begin{tabular}{lc}
\hline Characteristic & $\mathrm{n}(\%)$ \\
\hline Median age, years & 66 (range, 21-89) \\
Gender & $124(57)$ \\
Male & $94(43)$ \\
Female & \\
Cancer type & $152(70)$ \\
Lung & $66(30)$ \\
Pancreatic & \\
Degree of weight loss & $98(45)$ \\
$>5 \%$ & $62(28)$ \\
$\leq 5 \%$ & $58(27)$ \\
None &
\end{tabular}

Human Cytokine 27-Plex Assay (Bio-Rad Laboratories, Hercules, CA, USA) and analyzed using Bio-Plex Manager 2.0 software (Bio-Rad Laboratories).

The following biomarkers were assayed: GDF-15, Activin A, eotaxin, fibroblast growth factor basic, granulocyte colony stimulating factor, granulocyte-macrophage colony-stimulating factor, interferon- $\gamma($ IFN- $\gamma)$, interleukin (IL)-10, IL-12 p70, IL-13, IL-17, IL-1 receptor $\alpha$ (IL-1R $\alpha$ ), IL-2, IL-4, IL-5, IL-6, IL-7, IL-8, IL-9, IFN- $\gamma$-induced protein-10 (IP-10), monocyte chemotactic protein-1, macrophage inflammatory protein-1 $\alpha$ (MIP-1 $\alpha)$, MIP-1 $\beta$, platelet-derived growth factor-BB (PDGF-BB), regulated on activation, normal $\mathrm{T}$ cell expressed and secreted (RANTES), tumor necrosis factor- $\alpha$, and vascular endothelial growth factor A (VEGFA). These markers were selected as they have previously been implicated in cancer-associated weight loss or inflammation.

Statistical analysis. All data analyses were performed using $\mathrm{R}$ (version 3.1) statistical software (https://www.r-project. org/). Data are summarized descriptively. As all analyses were considered hypothesis-generating, no adjustments were made for multiple testing; however, all analyses primarily focused on GDF-15. The Wilcoxon rank sum test was used to compare the concentration of GDF-15 and other biomarkers according to the degree of weight loss exhibited by patients. A Cox Proportional Hazards Model was constructed to examine the prognostic significance of circulating GDF-15 levels and clinical parameters in univariate and multivariate analyses. $\mathrm{P}<0.05$ was considered to indicate a statistically significant difference.

\section{Results}

Patient demographics. A total of 218 patients were enrolled during a 1-year period. A total of 152 patients exhibited incurable lung cancer and 66 patients exhibited incurable pancreatic cancer. Overall, $98(45 \%)$ patients reported $>5 \%$ weight loss, $62(28 \%)$ patients reported $\leq 5 \%$ weight loss and $58(27 \%)$ reported no weight loss. Patient demographics are shown in Table I. 
Table II. Median biomarker plasma concentrations in 152 lung cancer patients exhibiting various degrees of weight loss.

\begin{tabular}{|c|c|c|c|c|c|c|c|c|c|}
\hline \multirow[b]{2}{*}{ Biomarker } & \multicolumn{2}{|c|}{$\begin{array}{l}\text { Median biomarker } \\
\text { concentration (pg/ml) }\end{array}$} & \multirow[b]{2}{*}{ P-value } & \multicolumn{2}{|c|}{$\begin{array}{l}\text { Median biomarker } \\
\text { concentration }(\mathrm{pg} / \mathrm{ml})\end{array}$} & \multirow[b]{2}{*}{$\mathrm{P}$-value } & \multicolumn{2}{|c|}{$\begin{array}{l}\text { Median biomarker } \\
\text { concentration }(\mathrm{pg} / \mathrm{ml})\end{array}$} & \multirow[b]{2}{*}{ P-value } \\
\hline & $\begin{array}{l}\text { No weight } \\
\text { loss group } \\
(n=48)\end{array}$ & $\begin{array}{c}>5 \% \\
\text { weight loss } \\
\text { group }(n=56)\end{array}$ & & $\begin{array}{l}\text { No weight } \\
\text { loss group } \\
(\mathrm{n}=48)\end{array}$ & $\begin{array}{c}\quad \leq 5 \% \\
\text { weight loss } \\
\text { group }(n=48)\end{array}$ & & $\begin{array}{c}\leq 5 \% \\
\text { weight loss } \\
\text { group }(n=48)\end{array}$ & $\begin{array}{c}>5 \% \\
\text { weight loss } \\
\text { group }(n=56)\end{array}$ & \\
\hline GDF15 & 1000 & 2000 & $<0.0001$ & 1000 & 2000 & 0.006 & 2000 & 2000 & 0.929 \\
\hline Activin A & 317 & 702 & 0.002 & 318 & 540 & 0.008 & 540 & 702 & 0.224 \\
\hline IL-12 p70 & 43 & 84 & 0.011 & 43 & 47 & 0.679 & 47 & 84 & 0.038 \\
\hline VEGFA & 33 & 55 & 0.025 & 33 & 36 & 0.623 & 36 & 55 & 0.069 \\
\hline IL-1R $\alpha$ & 288 & 597 & 0.035 & 288 & 340 & 0.588 & 340 & 597 & 0.117 \\
\hline Eotaxin & 52 & 88 & 0.038 & 52 & 77 & 0.07 & 77 & 88 & 0.576 \\
\hline PDGF-BB & 294 & 444 & 0.04 & 294 & 332 & 0.525 & 332 & 444 & 0.177 \\
\hline IL-10 & 29 & 71 & 0.052 & 29 & 29 & 0.972 & 29 & 71 & 0.054 \\
\hline IL-7 & 19 & 29 & 0.058 & 19 & 19 & 0.878 & 19 & 29 & 0.081 \\
\hline IL-6 & 23 & 39 & 0.064 & 23 & 26 & 0.651 & 26 & 40 & 0.141 \\
\hline IL-2 & 36 & 64 & 0.072 & 36 & 39 & 0.776 & 39 & 64 & 0.139 \\
\hline TNF- $\alpha$ & 99 & 154 & 0.105 & 99 & 99 & 0.994 & 99 & 154 & 0.114 \\
\hline IL-17 & 143 & 215 & 0.110 & 143 & 139 & 0.882 & 139 & 215 & 0.119 \\
\hline G-CSF & 160 & 212 & 0.116 & 160 & 175 & 0.652 & 175 & 212 & 0.382 \\
\hline IL-13 & 28 & 39 & 0.117 & 28 & 25 & 0.529 & 25 & 39 & 0.040 \\
\hline IL-8 & 79 & 124 & 0.120 & 79 & 69 & 0.456 & 69 & 124 & 0.067 \\
\hline MIP- $1 \alpha$ & 11 & 14 & 0.131 & 11 & 10 & 0.399 & 10 & 14 & 0.047 \\
\hline IFN- $\gamma$ & 199 & 282 & 0.161 & 199 & 177 & 0.536 & 177 & 282 & 0.088 \\
\hline IL-4 & 8 & 10 & 0.192 & 8 & 7 & 0.514 & 7 & 10 & 0.105 \\
\hline IL-5 & 24 & 33 & 0.227 & 24 & 25 & 0.849 & 25 & 33 & 0.349 \\
\hline $\mathrm{FGFb}$ & 110 & 130 & 0.331 & 110 & 98 & 0.435 & 98 & 130 & 0.149 \\
\hline GM-CSF & 69 & 82 & 0.346 & 69 & 56 & 0.158 & 56 & 82 & 0.066 \\
\hline MIP-1 $\beta$ & 139 & 97 & 0.375 & 139 & 83 & 0.238 & 83 & 97 & 0.299 \\
\hline MCP-1 & 97 & 115 & 0.380 & 97 & 83 & 0.261 & 83 & 115 & 0.136 \\
\hline IL-9 & 23 & 30 & 0.407 & 23 & 18 & 0.434 & 18 & 30 & 0.082 \\
\hline IP-10 & 285 & 312 & 0.443 & 286 & 281 & 0.861 & 281 & 312 & 0.273 \\
\hline RANTES & 13895 & 13798 & 0.976 & 13895 & 8938 & 0.057 & 8938 & 13799 & 0.059 \\
\hline
\end{tabular}

For RANTES, IL-2, IL-5 and IL-7 the sample size was smaller than the values stated due to assay detection issues. GDF-15, growth differentiating factor-15; IL, interleukin; VEGFA, vascular endothelial growth factor A; IL-1R $\alpha$, interleukin-1 receptor $\alpha$; PDGF-BB, platelet-derived growth factor-BB; TNF- $\alpha$, tumor necrosis factor- $\alpha$; G-CSF, granulocyte colony-stimulating factor; MIP-1 $\alpha$, macrophage inflammatory protein-1 $\alpha$; IFN- $\gamma$, interferon- $\gamma$; FGFb, basic fibroblast growth factor; GM-CSF, granulocyte-macrophage colony-stimulating factor; MIP- $1 \beta$, macrophage inflammatory protein-1 $\beta$; MCP-1, monocyte chemoattractant protein-1; IP-10, interferon- $\gamma$-induced protein-10; RANTES, regulated on activation, normal T cell expressed and secreted.

Association between weight loss and the concentration of GDF-15 and other biomarkers. Among the 152 lung cancer patients, statistically significant differences in GDF-15, Activin A, IL-12, VEGFA, IL-1R $\alpha$, eotaxin and PDGF-BB levels were observed between those who exhibited no weight loss and those who reported weight loss of $>5 \%$ (Table II), whereby increased weight loss was associated with higher concentrations of the circulating biomarkers. Upon comparison of lung cancer patients with no weight loss and those exhibiting weight loss of $\leq 5 \%$, statistically significant differences in levels of GDF-15 and Activin A were identified between the groups, whereby higher concentrations of GDF-15 and Activin A were identified in the $\leq 5 \%$ weight loss group compared with the no weight loss group $(\mathrm{P}=0.006$ and $\mathrm{P}=0.008$, respectively). Notably, significantly higher circulating IL-12 and IL-13 concentrations were identified in the
$>5 \%$ weight loss group when compared with the $\leq 5 \%$ weight loss group ( $\mathrm{P}=0.038$ and $\mathrm{P}=0.040$, respectively; Table II).

In pancreatic cancer patients, differences in biomarker concentrations between the groups were less evident. Only statistically significant differences in levels of the IP-10 biomarker were identified between the different weight loss groups. Specifically, patients in the $>5 \%$ weight loss group exhibited significantly higher circulating concentrations of IP-10 compared with patients that exhibited no weight loss $(\mathrm{P}=0.035$; Table III).

Higher GDF-15 concentration is associated with poor survival and increased weight loss. Univariate and multivariate analyses revealed that higher circulating concentrations of GDF-15 were associated with worse survival. Furthermore, higher circulating concentrations 
Table III. Median biomarker plasma concentrations in 66 pancreatic cancer patients exhibiting various degrees of weight loss.

\begin{tabular}{|c|c|c|c|c|c|c|c|c|c|}
\hline \multirow[b]{2}{*}{ Biomarker } & \multicolumn{2}{|c|}{$\begin{array}{c}\text { Median } \\
\text { biomarker } \\
\text { concentration }(\mathrm{pg} / \mathrm{ml})\end{array}$} & \multirow[b]{2}{*}{ P-value } & \multicolumn{2}{|c|}{$\begin{array}{c}\text { Median } \\
\text { biomarker } \\
\text { concentration }(\mathrm{pg} / \mathrm{ml})\end{array}$} & \multirow[b]{2}{*}{ P-value } & \multicolumn{2}{|c|}{$\begin{array}{c}\text { Median } \\
\text { biomarker } \\
\text { concentration }(\mathrm{pg} / \mathrm{ml})\end{array}$} & \multirow[b]{2}{*}{ P-value } \\
\hline & $\begin{array}{l}\text { No weight } \\
\text { loss group } \\
(\mathrm{n}=48)\end{array}$ & $\begin{array}{l}>5 \% \text { weight } \\
\text { loss group } \\
(n=56)\end{array}$ & & $\begin{array}{l}\text { No weight } \\
\text { loss group } \\
(\mathrm{n}=48)\end{array}$ & $\begin{array}{c}\leq 5 \% \text { weight } \\
\text { loss group } \\
(n=48)\end{array}$ & & $\begin{array}{c}\leq 5 \% \text { weight } \\
\text { loss group } \\
(n=48)\end{array}$ & $\begin{array}{l}>5 \% \text { weight } \\
\text { loss group } \\
(n=56)\end{array}$ & \\
\hline IP-10 & 263 & 471 & 0.035 & 263 & 406 & 0.072 & 406 & 471 & 0.551 \\
\hline PDGF-BB & 262 & 404 & 0.324 & 262 & 380 & 0.518 & 380 & 404 & 0.881 \\
\hline IL-17 & 143 & 242 & 0.363 & 143 & 178 & 0.729 & 178 & 242 & 0.487 \\
\hline IL-13 & 35 & 24 & 0.368 & 35 & 31 & 0.769 & 31 & 24 & 0.539 \\
\hline Activin A & 637 & 888 & 0.391 & 637 & 725 & 0.780 & 725 & 888 & 0.464 \\
\hline RANTES & 9806 & 12526 & 0.431 & 9806 & 9101 & 0.796 & 9101 & 12526 & 0.317 \\
\hline IL-5 & 62 & 28 & 0.495 & 62 & 24 & 0.457 & 24 & 28 & 0.551 \\
\hline GDF-15 & 2000 & 2000 & 0.504 & 2000 & 3000 & 0.196 & 3000 & 2000 & 0.405 \\
\hline MCP-1 & 126 & 86 & 0.508 & 126 & 84 & 0.492 & 84 & 86 & 0.936 \\
\hline IFN- $\gamma$ & 309 & 193 & 0.571 & 309 & 199 & 0.592 & 199 & 193 & 0.906 \\
\hline IL-6 & 58 & 34 & 0.604 & 58 & 31 & 0.563 & 31 & 34 & 0.762 \\
\hline Eotaxin & 69 & 88 & 0.626 & 69 & 75 & 0.883 & 75 & 88 & 0.592 \\
\hline IL-8 & 130 & 98 & 0.680 & 130 & 84 & 0.551 & 84 & 98 & 0.604 \\
\hline IL-7 & 32 & 25 & 0.745 & 32 & 19 & 0.558 & 19 & 25 & 0.232 \\
\hline IL-1R $\alpha$ & 558 & 419 & 0.759 & 558 & 285 & 0.543 & 285 & 419 & 0.305 \\
\hline IL-4 & 9 & 8 & 0.775 & 9 & 8 & 0.853 & 8 & 8 & 0.819 \\
\hline IL-9 & 26 & 30 & 0.818 & 26 & 25 & 0.961 & 25 & 30 & 0.614 \\
\hline MIP-1 $\beta$ & 120 & 111 & 0.828 & 120 & 122 & 0.961 & 122 & 111 & 0.782 \\
\hline TNF- $\alpha$ & 155 & 132 & 0.832 & 155 & 111 & 0.679 & 111 & 132 & 0.556 \\
\hline $\mathrm{FGFb}$ & 112 & 122 & 0.852 & 112 & 110 & 0.979 & 110 & 122 & 0.693 \\
\hline IL-12 p70 & 114 & 133 & 0.861 & 114 & 61 & 0.559 & 61 & 133 & 0.248 \\
\hline G-CSF & 233 & 257 & 0.874 & 233 & 189 & 0.759 & 189 & 257 & 0.385 \\
\hline MIP-1 $\alpha$ & 15 & 14 & 0.895 & 15 & 12 & 0.741 & 12 & 14 & 0.695 \\
\hline VEGFA & 55 & 60 & 0.915 & 55 & 55 & 0.995 & 55 & 60 & 0.844 \\
\hline IL-10 & 55 & 51 & 0.933 & 55 & 40 & 0.702 & 40 & 51 & 0.391 \\
\hline IL-2 & 56 & 53 & 0.939 & 56 & 41 & 0.685 & 41 & 53 & 0.526 \\
\hline GM-CSF & 83 & 81 & 0.976 & 83 & 56 & 0.455 & 56 & 81 & 0.127 \\
\hline
\end{tabular}

For RANTES, IL-2, IL-5 and IL-7 the sample size was smaller than that stated due to assay detection issues. IP-10, interferon- $\gamma$-induced protein-10; PDGF-BB, platelet-derived growth factor-BB; IL, interleukin; RANTES, regulated on activation, normal T cell expressed and secreted; GDF-15, growth differentiating factor-15; MCP-1, monocyte chemoattractant protein-1; IFN- $\gamma$, interferon- $\gamma$; IL-1R $\alpha$, interleukin-1 receptor $\alpha$; MIP-1 $\beta$, macrophage inflammatory protein-1 $\beta$; TNF- $\alpha$, tumor necrosis factor- $\alpha$; FGFb, basic fibroblast growth factor; G-CSF, granulocyte colony-stimulating factor; MIP-1 $\alpha$, macrophage inflammatory protein- $\alpha$; VEGFA, vascular endothelial growth factor A; GM-CSF, granulocyte-macrophage colony-stimulating factor.

Table IV. Univariate and multivariate survival analyses of GDF-15 levels and clinical variables.

\begin{tabular}{|c|c|c|c|c|}
\hline \multirow[b]{2}{*}{ Variable $^{\mathrm{a}}$} & \multicolumn{2}{|c|}{ Univariate analysis } & \multicolumn{2}{|c|}{ Multivariate analysis } \\
\hline & $\begin{array}{c}\text { Hazard ratio } \\
(95 \% \mathrm{CI})\end{array}$ & P-value & $\begin{array}{c}\text { Hazard ratio } \\
(95 \% \mathrm{CI})\end{array}$ & P-value \\
\hline GDF-15 level & $1.13(1.02-1.23)$ & 0.016 & $1.13(1.02-1.24)$ & 0.03 \\
\hline Age & $1.00(0.99-1.02)$ & 0.622 & $0.99(0.98-1.01)$ & 0.71 \\
\hline Gender & $0.78(0.56-1.09)$ & 0.15 & $0.79(0.57-1.12)$ & 0.19 \\
\hline Cancer type & $1.16(0.81-1.64)$ & 0.41 & $0.96(0.66-1.38)$ & 0.84 \\
\hline Weight loss & $0.6(0.43-0.83)$ & 0.0026 & $0.62(0.45-0.87)$ & 0.006 \\
\hline
\end{tabular}

${ }^{a}$ The reference group for each variable included higher GDF-15 level, older age, lung cancer, male gender and $<5 \%$ weight loss (includes patients with no weight loss), respectively. GDF-15, growth differentiating factor-15; CI, confidence interval. 
of GDF-15 were also associated with increased weight loss (Table IV).

\section{Discussion}

The present translational study focused on cancer-associated weight loss in patients with incurable lung or pancreatic cancer and aimed to investigate the association between this and the biomarker GDF-15. The results revealed that higher GDF-15 concentrations were significantly associated with weight loss in incurable lung cancer patients. Furthermore, in lung cancer patients increased weight loss was associated with higher concentrations of the circulating biomarkers, Activin A, IL-12, VEGFA, IL-1R $\alpha$, eotaxin and PDGF-BB. Notably, no significant differences were identified between GDF-15 concentration and weight loss in pancreas cancer patients; however, this may be due to the smaller sample size of pancreatic cancer patients included in the study. Furthermore, in univariate and multivariate analyses, GDF-15 level was significantly associated with poor survival. Following adjustments for weight loss and other clinical variables, high circulating concentrations of GDF-15 were significantly associated with shorter survival. These findings suggest that GDF-15 is involved in cancer-associated weight loss, particularly in lung cancer patients, and that it may serve as either a marker or future therapeutic target to prevent weight loss and its adverse effects in cancer patients.

Furthermore, the findings of the present study are consistent with a number of previous studies. Three previous studies have reported significant associations between this biomarker and weight loss and/or survival (8-10). The aforementioned studies and the present study have collectively provided clinical and laboratory data from $>500$ cancer patients. These data consistently indicate that GDF-15 is associated with cancer-associated weight loss. Johnen et al (8) reported that weight-losing, tumor-bearing mice that had been treated with an antibody to GDF-15 demonstrated reversal of their weight loss (8). Similarly, Lerner et al (12) also investigated weight loss reversal using a human fibrosarcoma xenograft murine model known to exhibit high levels of circulating GDF-15 and tissue wasting. In this model, the administration of a humanized antibody, AV-380, resulted in the reversal of weight loss and restoration of normal body composition. Taken together, these studies indicate that the association between GDF-15 and weight loss is significant and thus, may be important clinically.

The aim of the present study was to further characterize GDF-15 as a biomarker and to assess therapeutic targets to palliate cancer-associated weight loss; however, with regard to the latter, the complexity of the pathobiology of cancer-associated weight loss and the relative lack of specificity of GDF-15 with regard to a specific disease entity must be considered (6). However, the data obtained in the present study are compelling and the clinical requirement for well-designed clinical trials is high. Thus, future clinical trials that investigate GDF-15 as a biomarker and assess the therapeutic function of GDF-15 modulation in the palliation of cancer-associated weight loss are required.

\section{Acknowledgements}

This study was supported by AVEO Pharmaceuticals (grant no. R01CA124614).

\section{References}

1. Del Fabbro E, Dalal S and Bruera E: Symptom control in palliative care - Part II: Cachexia/anorexia and fatigue. J Palliat Med 9: 409-421, 2006.

2. von Haelhling S and Anker SD: Treatment of cachexia: An overview of recent developments. Int J Cardiol 184: 736-742, 2015.

3. Penet MF and Bhujwalla ZM: Cancer cachexia, recent advances, and future directions. Cancer J 21: 117-122, 2015.

4. Morley JE, Thomas DR and Wilson MM: Cachexia: Pathophysiology and clinical relevance. Am J Clin Nutr 83: 735-743, 2006.

5. Kim A and Nemeth E: New insights into iron regulation and erythropoiesis. Current Opin Hematol 22: 199-205, 2015.

6. Corre J, Hébraud B and Bourin P: Concise review: Growth differentiating factor 15 in pathology: A clinical role? Stem Cells Transl Med 2: 946-952, 2013

7. Tsai VW, Macia L, Johnen H, Kuffner T, Manadhar R, Jørgensen SB, Lee-Ng KK, Zhang HP, Wu L, Marquis CP, et al: TGF-b superfamily cytokine MIC-1/GDF15 is a physiological appetite and body weight regulator. PLoS One 8: e55174, 2013.

8. Johnen H, Lin S, Kuffner T, Brown DA, Tsai VW, Bauskin AR, Wu L, Pankhurst G, Jiang L, Junankar S, et al: Tumor-induced anorexia and weight loss are mediated by the TGF-beta superfamily cytokine MIC-1. Nat Med 13: 1333-1340, 2007.

9. Vigano A, Lerner L, Tao N, et al: From bench to bedside: are cytokines still relevant biomarkers for staging cancer cachexia? Proceedings of the $104^{\text {th }}$ Annual Meeting of the AACR, 2013; Washington, DC. Philadelphia; abstract 4650; 2013.

10. Lerner L, Hayes T, Tao N, et al: Plasma growth differentiating factor-15 (GDF-15) and other inflammatory markers are associated with weight loss and poor prognosis in cancer patients. Proceedings of the 104th Annual Meeting of the AACR; 2013 Apr 6-10; Washington, DC. Philadelphia; abstract 3504; 2013.

11. Fearon K, Strasser F, Anker SD, Bosaeus I, Bruera E, Fainsinger RL, Jatoi A, Loprinzi C, MacDonald N, Mantovani G, et al: Definition and classification of cancer cachexia: An international consensus. Lancet Oncol 12: 489-495, 2011.

12. Lerner L, Tao N, Krieger B, et al: Effective treatment of cancer associated cachexia by AV-380, a GDF15 inhibitory antibody. Proceedings of the 106th Annual Meeting of the AACR; 2015 Apr 18-22; Philadelphia, Pennsylvania; abstract 1115; 2015. 